\title{
Einfluß der Temperatur auf den Atmungsstoffwechsel der Puppen von Formica polyctena (Hymenoptera, Insecta)
}

\author{
GERHARD H. SCHMIDT \\ Institut für Angewandte Zoologie der Universität Würzburg, Würzburg
}

\begin{abstract}
Influence of temperature on the respiratory metabolism of pupae of Formica polyctena (Hymenoptera, Insecta). The influence of temperature on the respiratory processes of pupae of males, females and workers of the red wood ant Formica polyctena Foerst. has been investigated by means of the Warburg technique. At temperatures between $16^{\circ}$ and $32^{\circ} \mathrm{C}$ oxygen consumption values increase more or less along a straight line. Q10-values are reduced correspondingly, and in sexuals values reach below 1.0 at temperatures above $32^{\circ}$ or $34^{\circ} \mathrm{C}$. The curve then bends down. Between $16^{\circ}$ and $20^{\circ} \mathrm{C}, \mathrm{Q} 10^{-v}$ alues are above 5 . They are higher in sexuals than in workers. In addition to inactivation of enzymes at high temperatures, diffusion of oxygen is considered to limit respiration with increasing temperatures. The relation of metabolism versus body size of different castes has been analyzed using a double logarithmic plot. In sexual forms and workers weight proportionality of metabolism could be established. Respiratory quotients of different castes decline with decreasing temperature; they reach a maximum at the optimal temperature for development, i. e. $28^{\circ} \mathrm{C}$. The highest respiratory quotients as well as the most pronounced declines have been observed in workers. Undercooling leads to respiratory quotients close to 0.5 , revealing uncomplete oxidation at low temperatures. It is concluded that the activity of decarboxylases is strongly inhibited.
\end{abstract}

\section{EINLEITUNG}

Bei imaginalen Insekten und vielen anderen poikilothermen Tieren steigt die Atmungsintensität im Ruhezustand innerhalb des physiologischen Temperaturbereichs meistens exponentiell an, um dann nach Erreichen eines Maximalwertes wieder steil abzusinken. Auch der Rhythmus der Stigmen- und Pumpbewegungen wird mit zunehmender Temperatur beschleunigt (WIGgLESwORTH 1935, EDWARDS 1946). In einigen Fällen konnte bei der Optimaltemperatur ein Regulationsintervall festgestellt werden, wodurch die Exponentialkurve modifiziert wird (Precht, Christophersen \& Hensel 1955, SCHMID T 1956). Nach der vant Hoffschen RGT-Regel steigert eine Temperaturerhöhung um $10^{\circ} \mathrm{C}$ die Geschwindigkeit einer Reaktion um das 2- bis 3fache. Dies konnte im allgemeinen auch für die Insektenatmung beobachtet werden.

Bei Entwicklungsstadien verläuft die Atmung häufig nicht nach der RGT-Regel. Besonders während der Diapause ist der Atmungsstoff wechsel weniger empfindlich und steigt bei Temperaturerhöhung kaum an. Andere Verhälnisse findet man insbesondere während der Puppenphase der holometabolen Insekten, die jedoch bisher 
nur selten in vergleichende atmungsphysiologische Untersuchungen einbezogen worden ist.

Ein von der RGT-Regel abweichender Verlauf der Atmung konnte auch bei den hier untersuchten Formica-Puppen festgestellt werden. Diese Ameise bietet weiterhin die Möglichkeit, verschiedene Kasten im Temperaturgradienten vergleichend zu bearbeiten. Die in Abhängigkeit von der Temperatur gefundenen Stoffwechselkurven geben die Summe der Resultierenden von sehr vielen chemisch-physiologischen Reaktionen wieder, die durch eine Temperaturänderung verschieden beeinflußt werden können. Auf dieser Grundlage wurde versucht, den Atmungsstoffwechsel der FormicaPuppen in Abhängigkeit von der Temperatur zu analysieren.

\section{VERSUCHSTIERE UND METHODIK}

Alle Versuchstiere (Männchen, Weibchen und Arbeiterinnen) stammten aus vier Nestern von Formica polyctena FoERST. aus der Würzburger Umgebung. Alle Nester gehörten zu polykalen Kolonien. Die benötigten Puppen wurden während der Entwicklung im Frühjahr und Sommer aus den Nestern entnommen, von dem Kokongespinst befreit und nach Entwicklungsstadien sortiert, nachdem vorher die Entwicklung im Thermostaten verfolgt worden war. Für die hier aufgeführten Atmungsversuche wurden vorwiegend Puppen gewählt, die sich kurz vor dem Beginn der Körperausfärbung befanden. Dieses Stadium wird mit dem Terminus „Augen voll pigmentiert" gekennzeichnet (Scrmor 1964). Für alle weiteren untersuchten Entwicklungsstadien wurden prinzipiell die gleichen Ergebnisse erhalten. Alle Tiere werden jeweils vor dem Versuch gewogen und, wenn nicht anders vermerkt, vor den Versuchen 1 bis 2 Tage bei Zimmertemperatur $\left(20^{\circ}\right.$ bis $\left.22^{\circ} \mathrm{C}\right)$ gehalten.

Die Atmungsmessungen erfolgten mit Hilfe der Warburg-Technik. Die $\mathrm{Q}_{10}$-Werte wurden nach der Formel berechnet:

$$
\log Q_{10}=\frac{10}{T_{2}-T_{1}} \cdot \log \frac{R_{2}}{R_{1}}
$$

Hier bedeuten: $T_{2}-T_{1}=$ Temperaturdifferenz; $R_{2}$ und $R_{1}=$ Sauerstoff verbrauch bei $T_{2}$ beziehungsweise $T_{1}$.

Die bei Atmungsversuchen häufig zu beobachtende starke Streuung der Werte macht eine statistische Auswertung und Absicherung der Ergebnisse notwendig. Bei den verwendeten Puppen konnten die Schwankungen durch gezielte Auswahl gleichgroßer Tiere herabgesetzt werden. Obgleich stets 6 Geschlechtstiere beziehungsweise 15 Arbeiterinnen gleichzeitig getestet wurden, verlief die $\mathrm{CO}_{2}$-Abgabe immer unregelmäßiger als die $\mathrm{O}_{2}$-Aufnahme. Die maximale Streuung betrug zum Beispiel im 6stündigen Versuch bei zwölf 30-Minuten-Werten für den Sauerstoffverbrauch $5,8 \mathrm{~mm}^{3 / 6}$ oq $(14,6 \%)$ beziehungsweise $7,7 \mathrm{~mm}^{3 / 3}$ of $\hat{\delta}(38 \%)$. Die Berechnung der durchschnittlichen Streuung $\left(\eta= \pm \frac{\Sigma \mathrm{d}}{\sqrt{\mathrm{n}(\mathrm{n}-1)}}\right)$ ergab $\pm 3,3$ beziehungsweise $10,4 \%$ und der mittleren Abweichung $\left(\sigma= \pm \sqrt{\frac{\Sigma \mathrm{d}^{2}}{\mathrm{n}-1}}\right) \pm 4,2$ beziehungsweise $11,8 \%$. Für die 
$\mathrm{CO}_{2}$-Abgabe berechnete sich die maximale Streuung auf 27,5 bezichungsweise $47,4 \%$, die durchschnittliche Streuung auf $\pm 7,6$ bezichungsweise $11,4 \%$ und die mittlere $\mathrm{Ab}$ weichung auf $\pm 9,2$ beziehungsweise 13,9\%. Ahnliche Streuungen wurden bei allen derartigen respiratorischen Versuchen zwischen $16^{\circ}$ und $32^{\circ} \mathrm{C}$ gefunden. Diese für respiratorische Versuche ohnehin geringen Schwankungen wurden durch die Verwendung von $8 \times 6$ Geschlechtstieren beziehungsweise $8 \times 15$ Arbeiterinnen, woraus der definitive Mittelwert errechnet wurde, weiterhin stark reduziert. Für diese Mittelwerte errechnete sich die mittlere Streuung für den $\mathrm{O}_{2}$-Verbrauch auf $\pm 2 \%$, für die $\mathrm{CO}_{2}$ Abgabe auf $\pm 3 \%$; die vergleichbaren $R Q$-Werte zeigen eine mittlere Streung von $\pm 0,02$ Einheiten. Die Berechnung der Signifikanz erfolgte, soweit notwendig, mit Hilfe des t-Testes.

\section{ERGEBNISSE}

\section{Sauerstoffverbrauch und Körpergröße}

Die Messung des Sauerstoff verbrauchs von Individuen verschiedener Kastenzugehörigkeit und stark unterschiedlicher Größe bei verschiedenen Temperaturen machte es möglich, die Atmung der Puppen in Beziehung zur Körpergröße zu setzen. Eine kritische Analyse dieser Beziehungen gab Krüger (1961). Werden für einen interspezifischen Vergleich die unter konstanten Bedingungen ermittelten Werte für den Sauerstoffverbrauch in ein doppellogarithmisches Koordinatensystem in Abhängigkeit vom Körpergewicht aufgetragen, so verläuft die resultierende Gerade bei gestaltlich ähnlichen Insektenarten unter einem Winkel von meistens 30 bis $35^{\circ}$. Man spricht bei derartigen Winkelgrößen von einer Oberflächenproportionalität des Stoffwechsels (Bodine 1921, Konosumi 1934/35, Schmid 1956). Dies bedeutet jedoch lediglich, daß der Stoffwechsel proportional der Vergrößerung der Körperoberfläche ansteigt (RubNERsche Regel). Vergleicht man die Stoffwechselraten verschieden großer Imagines ein und derselben Art, so liegen die Winkelgrößen bei $45^{\circ}$. In diesem Falle wird von einer Gewichtsproportionalität des oxidativen Stoffwechsels gesprochen. Häufig wird eine Gerade gefunden, die noch steiler verläuft (KI'TTEL 1942, Mül. ER 1943, SCHMidt 1956).

Die Größe und damit das Gewicht der Imagines ist bei holometabolen Insekten weitgehend abhängig von der Menge der während der Larvenzeit gespeicherten Reservestoffe; aus großen Larven entwickeln sich große Imagines und umgekehrt aus kleinen Larven kleine Imagines. Es ist nicht bekannt, ob zwischen der Menge an gespeicherten Reservestoffen und der Ausbildung der imaginalen Atmungsfermente eine Beziehung besteht. In diesem Zusammenhang ist interessant, daß ELLENBY (1945) bei Vorpuppen von Drosopbila mit steigendem Gewicht einen Abfall im individuellen Sauerstoffverbrauch feststellte; auf die Oberfläche bezogen blieb der $\mathrm{O}_{2}$-Verbrauch gleich. Dagegen fand KNEITZ (1964) bei adulten Formica-Arbeiterinnen mit zunehmender Größe eine Erhöhung im Sauerstoffverbraud.

Eine Untersuchung verschiedener Kasten der sozialen Insekten hinsichtlich der Beziehung zwischen Stoffwechsel und Körpergröße ist bisher vernachlässigt worden. Aus Abbildung 1 ist ersichtlich, daß in dieser Hinsicht im untersuchten Temperatur- 
bereich stets eine deutliche Gewichtsproportionalität bei der hier untersuchten Art besteht. Inzwischen konnte KNErTZ (1964) diesen Befund für imaginale Tiere bestätigen. Damit behält die erwähnte innerartliche Beziehung auch für verschiedene Kasten

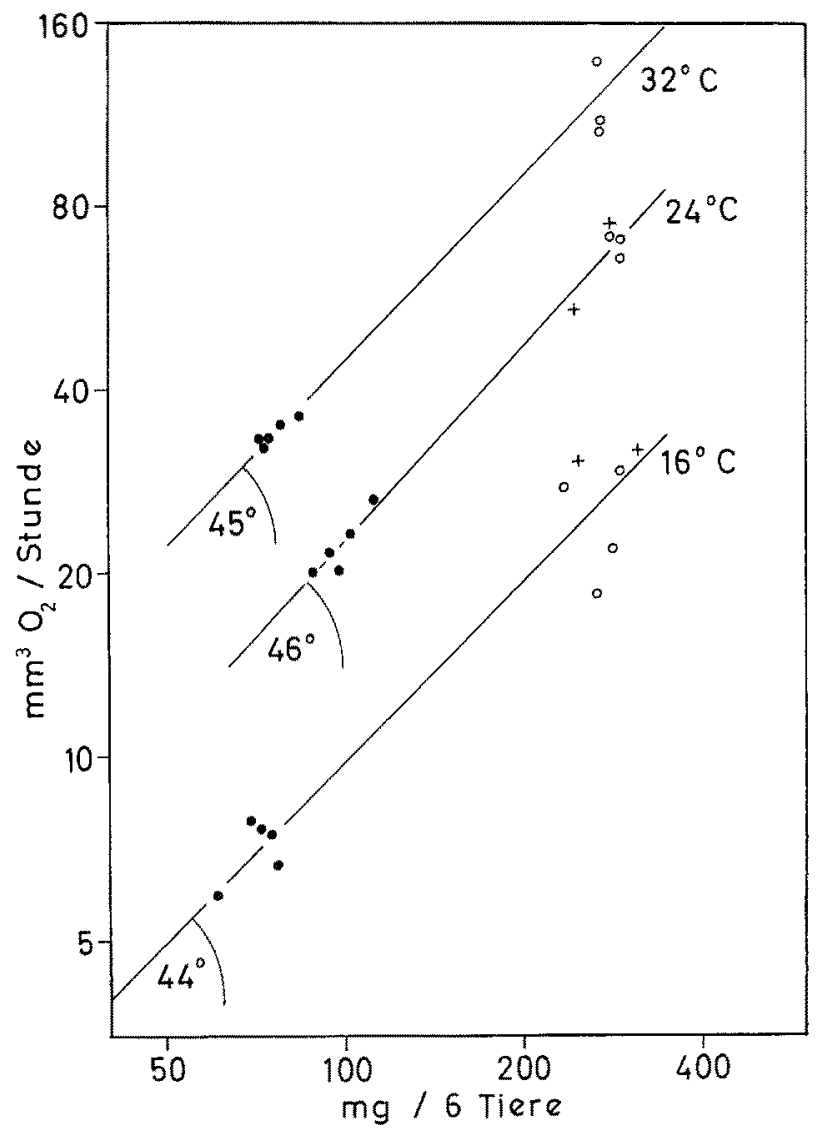

Abb. 1: Darstellung des Sauerstoffverbrauchs von Puppen (Stadium: Augen voll pigmentiert) bei verschiedenen Temperaturen als Funktion des Körpergewichts (nähere Angaben zur Berechnung bei KRÜGER 1958). Abszisse: Gewicht; Ordinate: Sauerstoffverbrauch bei den jeweils angegebenen Temperaturen; + Männdhen, 0 Weibchen, - Arbeiterinnen. Jeder Punkt ist ein Mittelwert von 6 Geschlechtstieren beziehungsweise 15 Arbeiterinnen, die jeweils uber 4 bis 5 Stunden untersucht wurden

sozialer Hymenopteren ihre Gültigkeit. Der Stoffwechsel der im Habitus abweichenden Männchen, welche etwa die Größe der Vollweibchen besitzen, entspricht nahezu dem der Weibchen.

\section{Temperaturabhängigkeit des Sauerstoffverbrauchs}

Die Messung des Sauerstoffverbrauchs in Abhängigkeit von der Temperatur erfolgte zwischen $16^{\circ}$ und $40^{\circ} \mathrm{C}$. Für die getesteten 48 Männchen, 48 Weibchen und 120 
Arbeiterinnenpuppen wurde der $\mathrm{O}_{2}$-Verbrauch in $2^{0}$-C-Intervallen nacheinander ermittelt. $\mathrm{Da}$ bei den einzelnen Temperaturstufen immer dieselben Tiere (jeweils Gruppen von 6 Geschlechtstieren beziehungsweise 15 Arbeiterinnen) untersucht wurden, ist ein direkter Vergleich möglich. Es wurde mit $16^{\circ} \mathrm{C}$ begonnen und dann die Temperatur alle 2 Stunden um $2^{\circ} \mathrm{C}$ erhöht. Nach einer halbstündigen Anpassung der Puppen an die Versuchstemperatur wurden für jede Temperaturstufe drei 30-Minuten-Werte gemessen und daraus der Mittelwert für den Sauerstoffverbrauch bei der betreffenden Temperatur errechnet.

Für die untersuchten Ameisenpuppen ergaben sich im Temperaturbereich zwischen $16^{\circ}$ bis $32^{\circ} \mathrm{C}$ für den Sauerstoffverbrauch allgemein abfallende $Q_{10^{-}}$Werte.

Tabelle 1

Q10-Werte für den Sauerstoffverbrauch von Männchen, Weibchen und Arbeiterinnen von $F$.polyctena bei Temperaturen zwischen $16^{\circ}$ und $32^{\circ} \mathrm{C}$; Puppenstadium: Augen voll pigmentiert

\begin{tabular}{|lcccccccc|}
\hline Geschlecht & $16-18^{\circ} \mathrm{C}$ & $18-20^{\circ} \mathrm{C}$ & $20-22^{\circ} \mathrm{C}$ & $22-24^{\circ} \mathrm{C}$ & $24-26^{\circ} \mathrm{C}$ & $26-28^{\circ} \mathrm{C}$ & $28-30^{\circ} \mathrm{C}$ & $30-32^{\circ} \mathrm{C}$ \\
\hline Männchen & 4,39 & 3,57 & 3,79 & 3,04 & 2,11 & 2,69 & 1,64 & 1,36 \\
Weibchen & 5,48 & 5,29 & 4,08 & 2,86 & 2,08 & 2,79 & 1,62 & 1,93 \\
Arbeiterinnen & 3,44 & 2,85 & 3,28 & 2,93 & 2,64 & 1,85 & 1,84 & 1,75 \\
\hline
\end{tabular}

Dementsprechend verlaufen die Sauerstoffverbrauchskurven in Abhängigkeit von der Temperatur nicht als typische Exponentialkurven (vergleiche hierzu PRECHT 1949), sondern als sehr schwache S-Kurven, die oberhalb $32^{\circ}$ beziehungsweise $34^{\circ} \mathrm{C}$ abknicken. Bei Temperaturen oberhalb $32^{\circ} \mathrm{C}$ sinken die $\mathrm{Q}_{10}$-Werte auf 1 und darunter ab (vergleiche hierzu auch die allgemeinen Ausführungen von Potonié 1924 und RAO \& Bullock 1954).

Auf der Suche nach einer Erklärung für das Absinken der $\mathrm{Q}_{10}-$ Werte mit steigender Temperatur mußte daran gedacht werden, daß nicht eine chemische Reaktion, sondern die Diffusion des Sauerstoffs in den Tracheen beziehungsweise Tracheolen für den Gesamtverlauf der Reaktionskette bestimmend ist. Weiterhin ist an eine allmähliche Inaktivierung von Fermenten mit steigender Temperatur zu denken. Die Untersuchung der Diffusionsverhältnisse ergab, daß während der Metamorphose keine Ventilations- und Zirkulationsbewegungen beobachtet werden können (SCHMIDT 1966). Daher erscheint die Annahme berechtigt, daß die Diffusionsgeschwindigkeit mit zunehmender Temperatur immer mehr zum kritischen Faktor wird und schließlich zu einer schwach S-förmigen Atmungskurve führt. Eine solche Situation würde allerdings ein Absinken der $\mathrm{Q}_{10}$-Werte unter 1 - wie es bei Geschlechtstieren über $32^{\circ} \mathrm{C}$ beziehungsweise über $34^{\circ} \mathrm{C}$ zu beobachten ist - nicht erklären können. (Bei Arbeiterinnen werden $\mathrm{Q}_{10^{-}}$Werte unter 1 bis hinauf zu Temperaturen von $40^{\circ} \mathrm{C}$ nicht festgestellt.) Möglicherweise wird dieser Abfall durch Ferment-Inaktivierung verursacht.

Auffallend sind auch die besonders bei Geschlechtstieren zu findenden sehr hohen $\mathrm{Q}_{10}$-Werte bei tiefen Temperaturen. Es kann angenommen werden, daß bei tiefen Temperaturen der Sauerstoffverbrauch durch eine fermentative, stark temperaturabhängige Teilreaktion gesteuert wird, die eine große Aktivierungsenergie benötigt, und daß diese Reaktion mit steigender Temperatur durch den Diffusionsprozeß beein- 
flußt wird, bis schließlich oberhalb von $32^{\circ} \mathrm{C}$ eine Inaktivierung einer am Atmungsprozeß beteiligten Teilreaktion eintritt, wodurch die Sauerstoffaufnahme herabgesetzt wird. Bei dieser Erklärungsmöglichkeit muß aber auch eine Disharmonisierung der funktionellen Integration des Organismus in Betracht gezogen werden. So könnte das Gefüge des Organismus durch einen Temperaturwechsel derart verändert werden, daß die einzelnen Entwicklungsprozesse verschiedene $Q_{10}$-Werte aufweisen und daraus dann unterschiedliche Gesamt-Q ${ }_{10}$-Werte resultieren. Derartige Veränderungen können sogar in verschiedenen Partien eines Organs auftreten (Hirsch 1929). Auf diese Weise vermag die Zuchttemperatur auch die Körperform zu becinflussen. Nach RAo \& BuLLOCK (1954) können sich die Q10-Werte mit der Größe der Tiere und der Adaptationstemperatur ändern.

\section{Einfluß niedriger Temperaturen}

Vergleicht man die Atmungsintensität von Puppen, welche Temperaturen zwischen $20^{\circ}$ und $22^{\circ} \mathrm{C}$ ausgesetzt worden waren, mit derjenigen von Puppen, die ein bis zwei Tage bei $6^{0} \mathrm{C}$ gehalten wurden, so ergibt sich eine deutlich e rhöhte Kohlendioxidabgabe bei Versuchsbeginn. Eine Versuchstemperatur von $24^{\circ} \mathrm{C}$ verursacht bei den an "normale" Temperaturen (Zimmertemperatur: $20^{\circ}$ bis $22^{\circ} \mathrm{C}$ ) adaptierten Puppen gleich zu Beginn der Versuche eine $\mathrm{CO}_{2}$-Abgabe, welche innerhalb der üblichen Grenzen praktisch konstant bleibt; die vorher bei $6^{\circ} \mathrm{C}$ gehaltenen Puppen dagegen geben in den ersten 30 Minuten doppelt soviel $\mathrm{CO}_{2} \mathrm{ab}$; bei ihnen werden die Normalwerte erst nach 1 bis 2 Stunden erreicht, obgleich bis zur Zeit der ersten Messung bereits eine Vorperiode von 45 Minuten vergangen war, während der aus methodischen Gründen nicht gemessen werden konnte. Im Gegensatz zur $\mathrm{CO}_{2}$-Abgabe veränderte sich der $\mathrm{O}_{2}$-Verbrauch nach der vorangegangenen Kältebehandlung nicht (Tab. 2).

Für jeden Einzelversuch wurden entweder 6 Geschlechtstier- oder 15 Arbeiterinnenpuppen ohne Kokon gleichen Stadiums und etwa gleicher Größe verwendet. Unter diesen Bedingungen konnten die auftretenden Gewichtsdifferenzen vernachlässigt werden. $\mathrm{O}_{2}$ - und $\mathrm{CO}_{2}$-Bestimmungen wurden mit den gleichen Tieren stets am gleichen Tage durchgefuhrt; dabei wurde abwechselnd zuerst das abgegebene Kohlendioxid oder der aufgenommene Sauerstoff ermittelt. Zur Prüfung des zeitlichen Verlaufs der Atmung wurden Versuche über 4 bis 6 Stunden durchgeführt. Halbstündlich wurde der Manometerstand ermittelt und die Gasaufnahme beziehungsweise Gasabgabe berechnet. Die genannte Tierzahl reichte aus, um reproduzierbare Durchschnittswerte zu erhalten.

Eine derartige, durch vorherige Unterkühlung verstärkte $\mathrm{CO}_{2}$-Abgabe konnte auch bei imaginalen $F$, polyctena-Weibchen festgestellt werden, bei denen sie den Flügelabwurf stimuliert (Gösswald \& SCHMIDT 1960). Bereits DREYER (1932), der eine $\mathrm{CO}_{2}$-Retention bei imaginalen Formica-Arbeiterinnen beobachtete, verwirft die Hypothese, daß die $\mathrm{CO}_{2}$-Retention allein durd die bei tiefen Temperaturen vermehrte physikalische Löslichkeit des Kohlendioxids bedingt sei (BETEL et al. 1960); auch die bei tiefen Temperaturen (beispielsweise $4^{\circ} \mathrm{C}$ ) gefundene, lang anhaltende, verminderte 
Tabelle 2

Zeitlicher Verlauf der $\mathrm{O}_{2}$-Aufnahme und $\mathrm{CO}_{2}-\mathrm{Abgabe}$ bei verschieden adaptierten Entwidklungsstadien von $F$. polyctena. Die Vorperiode zur Adaptation der Tiere an die Versuchstemperatur von $24^{\circ} \mathrm{C}$ betrug jeweils 45 Minuten, der Versuchsraum der Warburggefäße 15 bis $20 \mathrm{~cm}^{3}$

\begin{tabular}{|c|c|c|c|c|}
\hline \multirow[t]{2}{*}{$\begin{array}{l}\text { Versuchs- } \\
\text { zeit } \\
\text { (min) }\end{array}$} & \multicolumn{2}{|c|}{$\begin{array}{l}\text { Normal adaptiert }\left(20^{\circ}-22^{\circ} \mathrm{C}\right) \\
\text { Entw.-Stadium: vor der Imaginal- } \\
\text { häutung; Gewicht: } 157,4 \mathrm{mg}\end{array}$} & \multicolumn{2}{|c|}{$\begin{array}{c}\text { Kühl adaptiert }\left(6^{0} \mathrm{C}\right) \\
\text { Entw.-Stadium: Augen roll } \\
\text { pigmentiert; Gewicht: } 122,3 \mathrm{mg}\end{array}$} \\
\hline & $\mathrm{mm}^{3} / \mathrm{O}_{2} / 699$ & $\mathrm{~mm}^{3} / \mathrm{CO}_{2} / 6$ 9 9 & $\mathrm{~mm}^{3} / \mathrm{O}_{3} / 3 \partial \hat{\sigma}$ & $\mathrm{mm}^{3} / \mathrm{CO}_{2} / 3 \partial \partial$ \\
\hline $0-30$ & 42,0 & 37,8 & 24,8 & 37,1 \\
\hline $30-60$ & 38,7 & 36,2 & 17,9 & 25,8 \\
\hline $60-90$ & 37,0 & 29,4 & 17,1 & 18,9 \\
\hline $90-120$ & 39,5 & 33,6 & 23,1 & 25,7 \\
\hline $120-150$ & 38,7 & 31,1 & 18,7 & 18,7 \\
\hline $150-180$ & 39,5 & 28,5 & 21,3 & 21,3 \\
\hline $180-210$ & 42,8 & 35,2 & 23,1 & 19,6 \\
\hline $210-240$ & 41,2 & 37,0 & 18,7 & 17,8 \\
\hline $240-270$ & 40,3 & 37,9 & 19,6 & 22,2 \\
\hline $270-300$ & 38,7 & 36,2 & 21,3 & 22,2 \\
\hline $300-330$ & 39,5 & 34,4 & 17,9 & 16,1 \\
\hline $330-360$ & 41,2 & 33,6 & 19,6 & 18,7 \\
\hline Mittelwerte: & $39,7 \pm 1,65$ & $34,2 \pm 3,14$ & $20,3 \pm 2,44 *$ & $20,1 \pm 2,79^{*}$ \\
\hline
\end{tabular}

$\mathrm{CO}_{2}$-Abgabe schließt diese Hypothese aus. Bei einer Versuchstemperatur von $24^{\circ} \mathrm{C}$ sollte physikalisch gelöstes Kohlendioxid in wesentlich kürzerer Zeit entweichen, denn die Körpertemperatur hat sich in wenigen Minuten an die Versuchstemperatur angeglichen. Auch durch eine zweistundige Lagerung der Puppen in $90 \% \mathrm{CO}_{z}$-Atmosphäre konnte der Effekt einer $\mathrm{CO}_{2}$-Retention nicht erzielt werden. Vermutlich wird die Endoxidation gehemmt, indem die Dekarboxylierung verringert wird. Es werden Metaboliten angereichert, die bei höheren Temperaturen leichter decarboxyliert werden. Je länger die Puppen bei tiefen Temperaturen gehalten werden, um so höher ist anfänglich bei hohen Temperaturen die CO2-Abgabe (Unterkühlungszeiten 12 bis 96 Stunden). Die Retention des Kohlendioxids wird bei höheren Versuchstemperaturen schneller ausgeglichen als bei tieferen.

\section{Temperaturabhängigkeit des Respiratorischen Quotienten}

Nach den Ergebnissen der Versuche mit unterkühlten Tieren war zu erwarten, daß sich mit der Retention des Kohlendioxids auch die respiratorischen Quotienten als Funktion der Temperatur ändern. Während für Formica im Optimum Werte um 0,9 ermittelt wurden, erhielt bereits DrEYER (1932) bei Versuchstemperaturen von $4^{0}$ bis $6^{0} \mathrm{C}$ RQ-Werte von 0,5 bis 0,6 .

Allgemein werden mit sinkender Temperatur die Atmungsbewegungen der Insek- 
ten verlangsamt, was nicht nur durch den verminderten Sauerstoffverbrauch, sondern auch durch die nun stärker auftretende diskontinuierliche Kohlendioxidabgabe zum Ausdruck kommt (SchMid 1956). Da die Kohlendioxidproduktion in einem zwar wechselnden, jedoch bestimmten Verhältnis zum aufgenommenen Sauerstoff steht, muß sie mit der Verminderung der Oxydationsprozesse auch abnehmen. Dabei kann der RQ unverändert bleiben, wie dies CRescitelli (1935) für die Wachsmotte Galleria bei Temperaturen zwischen $20^{\circ}$ und $40^{\circ} \mathrm{C}$ feststellen konnte. Neben den erwähnten Untersuchungen von DrEYer (1932) fand aber auch KLEINMAN (1934) bei Heuschrecken mit abnehmender Versuchstemperatur ein Absinken der RQ-Werte, dessen Uriachen im einzelnen unbekannt blieben.

Zur Ermittlung der respiratorischen Quotienten der Formica-Puppen wurden Sauerstoffverbrauch und Kohlendioxidabgabe im Temperaturbereich von $16^{\circ}$ bis $32^{\circ} \mathrm{C}$ jeweils wenigstens über 4 Stunden bei jeder Versuchstemperatur nacheinander gemessen. Die Versuchstiere wurden täglich gewechselt. Bei jeder Temperaturstufe wurden etwa 50 Männchen-, 50 Weibchen- und 120 Arbeiterinnenpuppen untersucht und aus den erhaltenen Werten jeweils das Gesamtgruppenmittel errechnet, so daß für die respiratorischen Quotienten repräsentative Mittelwerte erhalten wurden. Um den Einfluß der Adaptationstemperatur weitgehend auszuschalten, wurden die Tiere vor jedem Versuch 2 Stunden im Warburggefäß an die jeweilige Versuchstemperatur adaptiert. Dies ist notwendig, da zu Versuchsbeginn vielfach erhöhte $\mathrm{CO}_{2}$-Abgabe stattfindet, die zu Verfälschungen der RQ-Werte führt. Nach zweistïndiger Adaptation erhält man gleichmäßige Werte.

Bei den hier untersuchten Puppen findet bereits zwischen $16^{\circ}$ und $32^{\circ} \mathrm{C}$ eine deutliche Reduktion der RQ-Werte mit sinkender Temperatur statt.

Tabelle 3

Respiratorische Quotienten von Männchen, Weibchen und Arbeiterinnen von $F$ : polyctena während der mittleren Puppenphase (Augen voll pigmentiert) in Abhängigkeit von der Temperatur

\begin{tabular}{|cccc|}
\hline${ }^{0} \mathrm{C}$ & Männchen & Arbeiterinnen & Weibchen \\
\hline 16 & 0,75 & 0,73 & 0,78 \\
20 & 0,77 & 0,87 & 0,72 \\
24 & 0,85 & 0,82 & 0,77 \\
28 & 0,92 & 0,94 & 0,86 \\
32 & 0,90 & 0,93 & 0,84 \\
\hline
\end{tabular}

Nach Thermostatversuchen liegt das Temperaturoptimum der Puppenentwicklung bei $28^{\circ} \mathrm{C}$. Die Temperaturspanne, in der überhaupt eine normale Entwicklung abläuft, ist relativ klein: $24^{\circ}$ bis $32^{\circ} \mathrm{C}$. Im Ameisennest wird diese Temperaturspanne durch Temperaturregulationen der Nestbevölkerung aufrechterhalten (STEINER 1925, 1947, Hermann 1963, Kneitz 1964).

Die RQ-Werte sinken besonders unterhalb des Optimums stark ab; oberhalb des Optimums tritt keine Erhöhung ein. Aus der Sauerstoffverbrauchskurve läßt sich jedoch entnehmen, daß die Entwicklungsstörungen oberhalb und unterhalb des Opti- 
mums verschiedener Natur sind. Bei den Arbeiterinnen, die im Optimum den höchsten RQ-Wert besitzen, ist die Reduktion mit abnehmender Temperatur am größten. Da nachgewiesen werden konnte, daß Arbeiterinnen während ihrer Puppenentwicklung von allen drei Kasten anteilmäßig am meisten Kohlenhydrate verbrauchen (SCHMIDT 1967), darf angenommen werden, daß sich die mit sinkender Temperatur auftretende Atmungsstörung besonders bei Kohlenhydratoxydation bemerkbar macht.

Das Absinken der RQ-Werte kann so gedeutet werden, daß infolge des sehr engen optimalen Temperaturbereichs bei ansonsten durchaus zuträglichen Temperaturen bereits eine Hemmung einer Dekarboxylase erfolgt, die bei $4^{\circ}$ bis $6^{\circ} \mathrm{C}$ nur noch verstärkt wird. Da das Optimum der Dekarboxylase-Wirkung offenbar sehr eng ist und hier mit dem optimalen Temperaturbereich zusammenfällt, kann vermutet werden, daß diese Dekarboxylase-Reaktion die Geschwindigkeit des oxydativen Endabbaues der Kohlenhydrate bestimmt, denn häufig ist die am langsamsten ablaufende Teilreaktion für den Gesamtverlauf verantwortlich. Es läßt sich die Hypothese ableiten, daß der optimale Temperaturbereich für die Entwicklung der Formica-Puppen nach unten durch die offenbar stark temperaturabhängige Reaktion einer Dekarboxylase festgelegt ist. Durch chemisch-analytische Untersuchungen sollte es möglich sein, das Substrat dieses Ferments näher zu charakterisieren.

\section{SCHLUSSBETRACHTUNG}

Auf Grund der besprochenen Befunde komme ich zu folgender Arbeitshypothese: Der steile Anstieg der $\mathrm{O}_{2}$-Verbrauchskurve bei tiefen Temperaturen deutet auf eine Teilreaktion hin, die eine hohe Aktivierungsenergie benötigt, damit die Gesamtreaktion ablaufen kann. Auf Grund der erhaltenen RQ-Werte könnte hiermit die Wirkung einer Dekarboxylase parallel gehen, die mit sinkender Temperatur stark reduziert wird. Mit zunehmender Temperatur wird der Kurvenlauf durch den Diffusionsprozeß mehr und mehr begrenzt, bis sich oberhalb des Optimums $\left(28^{\circ} \mathrm{C}\right)$ eine Inaktivierung von Fermenten bemerkbar macht und die Atmungskurve dann wieder abfällt. Denn bei adulten Tieren der gleichen Art, die Ventilationsbewegungen zeigen, erhält man nämlich eine typische Exponentialkurve, später aber auch einen Abfall der Kurve, der für Männchen bei $30^{\circ} \mathrm{C}$, für Weibchen und Arbeiterinnen bei $40^{\circ} \mathrm{C}$ beginnt (KNEITZ 1964).

Bei Zugrundelegung des vom Säugetier bekannten oxydativen Endabbaus kann die Dekarboxylase-Hemmung nicht die der Oxalbernsteinsäure oder $\alpha$-Ketoglutarsäure betreffen. Anderenfalls müßte im Unterkühlungsversuch bei Temperaturerhöhung eine - hier nicht gefundene - meßbare Steigerung des Sauerstoffverbrauchs auftreten. Dagegen ist eine Hemmung der Brenztraubensäure-Dekarboxylase denkbar, worauf vor allem auch die besonders stark absinkenden RQ-Werte der vermehrt Kohlenhydrat oxydierenden Arbeiterinnen hinweisen. Für die Oxydation der Kohlenhydrate bis zur Brenztraubensäure würde zwar Sauerstoff verbraucht, jedoch kein $\mathrm{CO}_{2}$ frei, wodurch die RQ-Werte reduziert würden. Die $Q_{10}$-Werte würden durch einen mit sinkender Temperatur sich immer stärker bemerkbar machenden Ausfall der Endoxydation der Kohlenhydrate erhöht. 


\section{ZUSAMMENFASSUNG}

1. Der Einfluß der Temperatur auf die Atmungsprozesse der Puppen von Männchen, Weibchen und Arbeiterinnen der Roten Waldameise Formica polyctena FoERST. wurde mit Hilfe der Warburg-Technik untersucht.

2. Die Sauerstoffyerbrauchskurve steigt zwischen $16^{\circ}$ und $32^{\circ} \mathrm{C}$ schwach S-förmig an. Die $Q_{10^{-}}$Werte nehmen entsprechend ab und erreichen bei Geschlechtstieren oberhalb $32^{\circ}$ beziehungsweise $34^{\circ} \mathrm{C}$ Werte unter 1 . Die Kurve knickt dann ab. Zwischen $16^{0}$ und $20^{\circ} \mathrm{C}$ wurden $\mathrm{Q}_{10^{-}}$Werte über 5 ermittelt. Sie liegen bei Geschlechtstieren höher als bei Arbeiterinnen.

3. Neben der Inaktivierung von Fermenten durch hohe Temperaturen wird die Diffusion des Sauerstoffs als der die Atmung begrenzende Prozeß angesehen.

4. Erstmals konnte im doppellogarithmischen Koordinatensystem die Beziehung zwischen Stoffwechsel und Körpergröße der Puppen verschiedener Kasten geprüft werden. Zwischen Geschlechtstier- und Arbeiterinnenpuppen wurde eine eindeutige Gewichtsproportionalität gefunden. Damit behält diese innerartliche Beziehung auch für verschiedene Kasten sozialer Hymenopteren ihre Gültigkeit.

5. Eine Untersuchung der RQ-Werte der Puppen verschiedener Kasten zeigte, daß diese mit sinkender Temperatur abfallen. Sie erreichten ihr Maximum bei der optimalen Entwicklungstemperatur von $28^{\circ} \mathrm{C}$. Die höchsten RQ-Werte wurden bei Arbeiterinnen festgestellt; hier zeigte sich auch der stärkste Abfall. Versuche bei tiefen Temperaturen lieferten RQ-Werte von 0,5. Dieser Umstand läßt darauf schließen, daß bei tiefen Temperaturen eine unvollständige Oxydation stattfindet.

6. Es wird vermutet, daß die Aktivität von Decarboxylasen mit sinkender Temperatur gehemmt wird.

\section{ZITIERTE LITERATUR}

Betel, J, Punt, A., Parser, W. \& Geest, H. van, 1960. COg-transport in insect haemolymph. Archs néerl. Zool. 13, 590-592.

Bopme, H. J., 1921. Factors influencing the water content and rate of metabolism of certain orthoptera. J. exp. Zool. 32, 137.

CRescrtelli, F., 1935. The respitatory metabolism of Galleria mellonella (bee moth) during pupal development at different constant temperatures. I. cell comp. Physiol. 6, 351-368.

DREYER, W. A., 1932. The effect of hibernating and seasonal variation of temperature on the respiratory exchange of Formica ulkei EM. Physiol. Zool. 5, 301-331.

EDWARDS, G. A., 1946. The influence of the temperature upon the oxygen consumption of several arthropodes. J. cell. comp. Physiol. 27, 53-64.

Ellenby, C., 1945. Oxygen consumption of prepupae of Drosophila melanogaster Meig., in relation to the surface area of the puparium. f. exp. Biol. 21, 39-45.

GösswaLD, K. \& SchMmT, G. H., 1960. Untersuchungen zum Begattungsverhalten und Flïgelabwurf einiger Formica-Arten im Hinblick auf ihre systematische Differenzierung. Insectes soc. $7,298-321$.

Hemann, M., 1963. Zum Warmehaushalt der Kleinen Roten Waldameise (Fomica polyctena Foerst.). Waldbygiene 5, 1-21.

Hirsce, G. Ch., 1929. Dynamik organischer Strukturen. Gedanken zur Methodik ihrer Untersuchung. Wilbeln Roux Arch. Entwe. Mech. Org. 117, 511-561. 
Krtтel, A., 1941. Körpergröße, Körperzeiten und Energiebilanz. 2. Der Sauerstoffverbrauch der Insekten in Abhängigkeir von der Körpergröße. Z. vergl. Physiol. 28, 533-662.

KLEINMAN, L. W., 1934. The effect of temperature upon the respiratory quotient of nymphs of the grasshopper, Chortophaga viridifasciata DE GEER, and larvae of the Japanese beetle, Popillia japonica NEWMAN, with reference to changes during hibernation. J. cell. comp. Physiol. 4, 221-235.

KNEITZ, G., 1964. Untersuchungen zum Aufbau und zur Erhaltung des Nestwärmehaushaltes bei Formica polyctena Foerster (Hym. Formicidae). Würzburg, Nat. math. Diss., 156 pp.

Korbsum, K., 1934/35. Experimentelle Studien über die Transpiration und den Wärmehaushalt der Insekten. Mem. Fac. Sci. Agric. Taiboku imp. Univ. 12, 1-179, 281-380.

Krǘger, F., 1958. Größenabhängigkeit des Sauerstoffverbrauchs einheimischer Grillen. Biol. Zbl. 77, 581-588.

- 1961. Ober die Exponenten dex Temperaturfunktion biologischer Vorgänge und deren Größenabhängigkeit. Biol. Zbl. 80, 721-750.

MülLLE, I., 1943. Untersuchungen über die Gesetzlichkeit des Wachstums. Die Abhängigkeit der Atmung von der Körpergröße bei Dixippus morosus und ihre Beziehungen zum Wachstum. Z. vergl. Physiol. 30, 139-144.

PotonIE, W. H., 1924. Experimentelle kritische Untersuchungen über die biologische Bedeutung des Umkehrpunktes in der Atmungsintensität kaltblütiger Tiere bei steigender Temperatur. Biol. Zbl. 44, 16-57.

Precht, H., 1949. Die Temperaturabhängigkeit von Lebensprozessen. Z. Naturf. 4b, 26-35.

- Christophersen, J. \& Hensel, H., 1955. Temperatur und Leben. Springer, Göttingen, 514 pp.

RaO, K. P. \& Bulzock, TH. H., 1954. Q10 as a function of size and habitat temperature in poikilotherms. Am. Nat. 88, 33-44.

Scrmmt, G. H., 1956. Der Stoff wechsel der Caraben (Ins. Coleopt.) und seine Beziehung zum Wasserhaushalt. Zool. Jb. (Allg. Zool. Physiol. Tiere) 66, 273-294.

- 1964. Histologische Untersuchungen zur Metamorphose des Mitteldarmepithels von Formica polyctena Foerster (Ins. Hym.). Biol. Zbl. 83, 717-724.

- 1966. Allometrien in der Ausbildung imaginaler Organe während der Metamorphose von Formica polyctena Foerster (Ins. Hym.). Biol. Zbl. 85, 137-158.

- 1967. Veränderungen im Gehalt an energieliefernden Reservestoffen während der Kastendifferenzierung von Formica polyctena Foerster. (Ins. Hym. Formicidae). Biol. Zbl. 86 (im Drudk).

Sterner, A., 1925. Uber def sozialen Wärmehaushalt der Roten Waldameise (Formica rufa, var. rufa pratensis For.). Z. vergl. Physiol. 2, 23-56.

- 1947. Der Wärmehaushalt der einheimischen sozialen Hautflügler (Wespen, Hummeln, Bienen und Ameisen). Beih. schweiz. Bienenztg 2, 139-256.

WigglesworTh, V. B., 1935. The regulation of respiration in the flea, Xenopsylla cheopis Roths. (Pulicidae). Proc. R. Soc. B 118, 397-419.

\section{Diskussion im Anschluß an den Vortrag ScHMID T}

KrüGER: Ich habe Bedenken gegen die Anwendung des Q10-Wertes, wenn er nicht konstant ist. In solchen Fällen muß man eine andere Form der Beschreibung versuchen, ohne hierbei auf meinem eigenen Vorschlag zu bestehen. Der Q10-Wert beruht auf der alten Formulierung von BeRTHELOT: $c=a \cdot b t$. Eine eindeutige Charakterisierung des Verlaufs von Temperaturkurven ergibt sich nur, wenn man die Parameter $a$ und $b$ angibt. $b$ ist die 10 . Wurzel von $Q_{10}$ und liefert ungeschickte Zahlen. In dieser Beziehung ist der Q10-Wert praktischer, hat aber nur einen Aussage-Wert, wenn ex konstant ist. In Ihrem Falle ist das nicht der Fall. Der Q10-Wert hat nur eine Bedeutung als Faustregel, kann aber nur in Ausnahmefällen bei biologischen Objekten zur Versuchsauswertung dienen.

Sснmid: Es war nicht das Ziel meiner Untersuchungen, für den Verlauf der Atmungskurven 
eine mathematische Formulierung zu finden, sondern nur den Verlauf der Kurve experimentell zu analysieren. Hierfür erscheinen mir die berechneten $Q_{10}$-Werte ausreichend.

RoBERTs: Have you an idea to help explain the relationship between body-size and metabolism in insects which is often reported (VON BERTALANFFY) to give very high "a" values close to unity.

Schmid: Die Beziehung zwischen Körpergröße und Stoffwechsel gibt nur Auskunft über das Verhältnis beider Größen, sagt aber nichts über die Stoffwechselart aus. Im vorliegenden Falle geht die Vermehrung der respiratorisch wirksamen Gewebe der Gewichtszunahme parallel.

WIESER: Wenn ich Ihre Tabelle richtig gelesen habe, scheint es mir, als ob die bei $6^{\circ} \mathrm{C}$ gelagerten und bei $24^{\circ} \mathrm{C}$ gemessenen Puppen einen wesentlich niedrigeren Sauerstoffverbrauch hatten, als die bei Zimmertemperatur gelagerten.

Schmidt: In Tabelle 2 sind verschiedene Entwicklungsstadien aufgeführt, was jedoch für die Ausführungen unwichtig ist. Jedes Stadium zeigt nach Unterkühlung bei $24^{\circ} \mathrm{C}$ eine erhöhte $\mathrm{CO}_{2}$-Abgabe, während der $\mathrm{O}_{2}$-Verbrauch weder in der Höhe noch im Verlauf bei vergleichbaren Stadien variiert; es tritt anfangs keine erhöhte $\mathrm{O}_{2}$-Aufnahme ein. Verschiedene Entwicklungsstadien können sich in der Höhe des $\mathrm{O}_{2}$-Verbrauchs natürlich unterscheiden. 